\title{
Reply to Brioude et al
}

\author{
Jack Brzezinski ${ }^{1}$ - Cheryl Shuman ${ }^{1}$ - Sanaa Choufani ${ }^{1} \cdot$ Peter Ray $^{1} \cdot$ Dimitri J Stavropoulos ${ }^{1} \cdot$ Raveen Basran $^{1}$. \\ Leslie Steele $^{1} \cdot$ Nicole Parkinson $^{1} \cdot$ Ronald Grant $^{1} \cdot$ Paul Thorner $^{1} \cdot$ Armando Lorenzo $^{1} \cdot$ Rosanna Weksberg $^{1}$
}

Received: 15 December 2017 / Accepted: 23 December 2017 / Published online: 15 February 2018

(c) European Society of Human Genetics 2018

We thank Brioude et al. for their interest in our report on children with Beckwith-Wiedemann Syndrome (BWS) who presented with Wilms tumors (WT) or preneoplastic lesions and an initial molecular diagnosis of loss of methylation at imprinting center 2 (IC2 LOM) [1]. We agree that IC2 LOM confers a lower risk for WT when compared with other BWS-associated molecular etiologies. However, defining which children with BWS should undergo tumor surveillance is a complex problem that, in our opinion, requires further investigation and discussion. We arrived at this position for a number of reasons including: (1) the challenges of molecular classification of BWS that impact accurate estimation of WT risk for each BWS subgroup; (2) the increasing number of WT in individuals with BWS and IC2 LOM that have come to our attention; (3) the relevance of hypertrophic nephrogenic rests to the development of WT; (4) the increased liability of individuals with BWS and IC2 LOM to develop end stage renal disease emphasizing the need for early detection of WT in this population.

The challenge of accurately determining the molecular diagnosis in BWS is especially relevant in considering revisions of tumor surveillance guidelines. Brioude et al. underscore the need for testing to be undertaken in an experienced laboratory. In fact, the cases in our report were assessed by a highly experienced clinical laboratory that functions as a national/international reference center with decades of experience in diagnostics of imprinting disorders. Moreover, the clinical testing employed was the most robust available at that point in time. For case 1, this included MS-MLPA and STR analysis which highlighted the fact that somatic mosaicism for paternal uniparental disomy (pUPD11p15.5) may lead to a situation where gain

Rosanna Weksberg

rweksb@sickkids.ca

The Hospital for Sick Children, Toronto, ON, Canada of methylation at the H19 DMR may not be detectable in accessible tissues such as blood.

Consideration of evolving testing approaches raises an important consideration in the estimation of tumor frequencies stratified by (epi)genotype. As noted by Brioude and others, SNP arrays can increase the accuracy of identifying the correct molecular etiology. Indeed, SNP array was undertaken as part of the extensive investigation after the development of WT in our case 1, supporting a diagnosis of UPD. However, this test modality was not available at the time of initial assessment. Therefore given the exigencies of BWS molecular testing, it should be expected that some children with low-level mosaicism for pUPD11p15.5will be misclassified as IC2 LOM. For the molecular analyses and meta-analysis of the participants in the Maas publication, this means that some patients used to inform the risk calculation may have been misclassified. Such misclassification would lead to an inflation of the number of patients diagnosed with IC2 LOM with a concomitant deflation in the number of patients with pUPD11p15.5. Since the majority of such misclassified cases would not present with WT, this would inflate the number of IC2 LOM cases without WT and lead to an inappropriate reduction in the risk estimate for WT in the LOM IC2 group. Similarly the WT risk for pUPD11p15.5 would be inflated. Accurate estimates of WT risk by BWS molecular subgroup therefore must await implementation of optimized molecular testing using multiple platforms that interrogate both SNPs and DNA methylation, and potentially include multiple tissues, as standard of care for all children with BWS. The implementation of such molecular testing should occur in the context of a broad prospective ascertainment of large numbers of children with BWS.

Since the Brzezinski publication, we have been informed of two additional cases of WT associated with LOM IC2 not as yet reported. Therefore, at least five children in addition to the five previously reported by Maas et al. [2], Ibrahim et al. [3], and Niemitz et al. [4] would not have been screened using the guidelines proposed by Maas and 
others. Additional cases will likely continue to surface especially given the recent advocacy for screening nonsyndromic children presenting with WT using MS-MLPA for $11 \mathrm{p} 15.5$ [5].

With respect to nephrogenic rests and our case 2, there is an important distinction between microscopic and hypertrophic rests. Many microscopic rests are considered dormant, developmental remnants that are only detected on pathologic investigations. In contrast, the development of hypertrophic nephrogenic rests are well-accepted WT precursors that may be visualized by imaging as was the case in our series [6]. The transition of nephrogenic rests from dormancy to preneoplastic lesions is likely driven by the accumulation of WT-associated molecular alterations [7]. Thus, the identification of such lesions in a child with BWS is of concern as these are clear signs of a high risk of developing WT. Spontaneous resolution of a preneoplastic lesion does not negate the existence of a predisposition to WT.

As Brioude et al. note, children with IC2 LOM demonstrate an increased risk of anatomical renal anomalies including renal cysts, nephrocalcinosis [8] and medullary sponge kidney reflecting the significant risk for renal dysfunction later in life, especially if there is substantial loss of renal mass due to late treatment of WT. Tumor surveillance for all children with BWS including those with LOM IC2 allows early detection of WT in this patient group thereby optimizing the likelihood that nephron-sparing surgery can be performed and reducing the chance that nephrotoxic abdominal radiation will be required at later stages of the disease. Data from the fourth National Wilms Tumour Study strongly suggest that a screening program increases the rate of early detection and a concomitant smaller size of tumor at the time of diagnosis $[9,10]$ thus increasing the likelihood of successfully sparing normal renal tissue.

While the meta-analysis published by Maas et al. [2] indicates a low prevalence (1/500) of WT in children with IC2 LOM when compared to children with BWS due to other etiologies, this prevalence is markedly higher than the $\sim 7.1 / 1000000$ prevalence in the general population of children under 15 years of age [11]. For many health care providers and parents, the significantly increased risk of WT above that of the general population is sufficient rationale to implement a screening strategy. The psychological impact of a tumor surveillance program is complex and while there is some evidence that screening increases anxiety in families, a recent study indicates that parents report that surveillance is not burdensome and that it decreases their worry overall [12].
We conclude that there is an increased risk of WT in children with BWS and LOM IC2 when compared to the general population, and that tumor surveillance aimed at early detection of such tumors is vitally important given the potential for long-term renal complications in these children. Based on these considerations and the challenges associated with current molecular testing methodologies in accurately defining BWS molecular etiology, there is, for us, sufficient rationale to continue to recommend tumor surveillance for all children with BWS. This approach ensures early diagnosis and targeted treatment to optimize the care and quality of life of children and adults with BWS.

\section{References}

1. Brzezinski J, Shuman C, Choufani S, et al. Wilms tumour in Beckwith-Wiedemann Syndrome and loss of methylation at imprinting centre 2: revisiting tumour surveillance guidelines. Eur J Hum Genet. 2017;25:1031-9.

2. Maas SM, Vansenne F, Kadouch DJ, et al. Phenotype, cancer risk, and surveillance in Beckwith-Wiedemann syndrome depending on molecular genetic subgroups. Am J Med Genet. 2016;170:2248-60.

3. Ibrahim A, Kirby $\mathrm{G}$, Hardy $\mathrm{Cu}$, et al. Methylation analysis and diagnostics of Beckwith-Wiedemann syndrome in 1,000 subjects. Clin Epigenetics. 2014;6:11.

4. Niemitz EL, Feinberg AP, Brandenburg SA, Grundy PE, DeBaun MR. Children with idiopathic hemihypertrophy and beckwithwiedemann syndrome have different constitutional epigenotypes associated with wilms tumour. Am J Hum Genet. 2005;77:887-91.

5. Mifsud, W \& Pritchard-Jones, K. Paediatrics: Integrating genomics to dig deeper into Wilms tumour biology. Nat Rev Urol. 2017. https://doi.org/10.1038/nrurol.2017.178

6. Beckwith JB, Kiviat NB, Bonadio JF. Nephrogenic rests, nephroblastomatosis, and the pathogenesis of Wilms' tumour. Pediatr Pathol. 1990;10:1-36. (1990)

7. Charlton J, Williams RD, Sebire NJ, et al. Comparative methylome analysis identifies new tumour subtypes and biomarkers for transformation of nephrogenic rests into Wilms tumour. Genome Med. 2015;7:11.

8. Goldman M, Smith A, Shuman C, et al. Renal abnormalities in beckwith-wiedemann syndrome are associated with $11 \mathrm{p} 15.5$ uniparental disomy. J Am Soc Nephrol. 2002;13:2077-84.

9. Porteus MH, Narkool P, Neuberg D, et al. Characteristics and outcome of children with Beckwith-Wiedemann syndrome and Wilms' tumour: a report from the National Wilms Tumour Study Group. J Clin Oncol. 2000;18:2026-31.

10. McNeil DE, Langer JC, Choyke P, DeBaun MR. Feasibility of partial nephrectomy for Wilms' tumour in children with BeckwithWiedemann syndrome who have been screened with abdominal ultrasonography. J Pediatr Surg. 2002;37:57-60.

11. SEER Review 1975-2011Childhood Cancer. 2014; 1-14.

12. Duffy KA, Grand KL, Zelley K, Kalish JM. Tumour screening in Beckwith-Wiedemann syndrome: parental perspectives. J Genet Couns. 2017;132:377. 